Case report

\title{
A 21-year-old man with late Wallenberg syndrome after a motorcycle trauma
}

\author{
Vitorino Modesto dos Santos ${ }^{1,2}$, Ursula Valeska Poti Araújo Lima ${ }^{2}$, Renata Faria Silva ${ }^{2}$, \\ Silvana Alves Gomma de Azevedo ${ }^{2}$, Melissa Gebrim Ribeiro ${ }^{2}$, Daniela Gomes Gebrim ${ }^{2}$ \\ ${ }^{1}$ Catholic University Medical Course, Brasília-DF, Brazil \\ ${ }^{2}$ Armed Forces Hospital, Brasília-DF, Brazil
}

Received 10 March 2015, Accepted 18 April 2015

(C) 2015, dos Santos V.M., Lima U.V.P.A., Silva R.F., de Azevedo S.A.G., Ribeiro M.G., Gebrim D.G.

(C) 2015, Russian Open Medical Journal

\begin{abstract}
Spontaneous and traumatic dissection of vertebral arteries can cause ischemic or hemorrhagic strokes in young individuals. Clinical features often propitiate a challenging diagnosis because initial presentations are variable, from asymptomatic till very ominous conditions. Therefore, an elevated index of suspicion and use of imaging resources might favor early diagnosis and prevention of serious consequences. We report a healthy 21-year-old man who had a motorcycle accident about fourty days before the imaging confirmation of vertebral artery dissection and thrombosis of the basilar artery. The patient successfully underwent non invasive management, with use of acetylsalicylic acid.
\end{abstract}

Keywords: stroke, vertebral artery dissection, vertebrobasilar ischemia

Cite as dos Santos VM, Lima UVPA, Silva RF, de Azevedo SAG, Ribeiro MG, Gebrim DG. A 21-year-old man with late Wallenberg syndrome after a motorcycle trauma. Russian Open Medical Journal 2015; 4: e0304.

Correspondence to Prof. Vitorino Modesto dos Santos, PhD. Address: Armed Forces Hospital, Estrada do Contorno do Bosque s/n, Cruzeiro Novo, 70658-900, Brasília-DF, Brazil. Phone: \#55-61 39662103. Fax: \#55-61 32331599. E-mail: vitorinomodesto@gmail.com

\section{Introduction}

Vertebral artery dissection (VAD) is one of the most frequent causes of stroke affecting people under 50 years of age, and up to $30 \%$ of the episodes are ischemic [1-3]. VAD may occur spontaneously, or is secondary to blunt trauma or neck movements; and the estimated incidence of spontaneous VAD is up to 3 per 100,000 per year $[1,2,4]$. This condition may evolve unsuspected and with poor outcomes, if imaging studies are not done to early diagnosis of the mechanisms of progressive neurological symptoms [1]. Undiagnosed thromboembolic episodes involving the basilar artery should be considered an ominous possibility in this setting [1]. Headache and neck pain are the most frequent initial symptoms of VAD, but the early clinical suspicion about this uncommon condition often constitutes a challenging task $[2,5,6]$. Although the prognosis of unruptured VAD can be ominous, there is no consensual guideline and respective therapeutic options remain controversial [5-7].

We present the case of a young Brazilian male with left VAD followed by thrombosis of the basilar artery, which were controlled with success by conservative management, approximately two months after a head and neck trauma related to a severe motorcycle accident.

\section{Case report}

A previously healthy 21-year-old man had been subjected to multiple traumatic injuries about 40 days prior to admission, involving the head and neck, in addition to a transverse fracture of left elbow. He came to our hospital due to headache nausea, vomiting, changes in gait and balance, abnormal speech, difficult to swallow, and tingling on his left hemiface, started 24 hours before receiving our medical attention. He had no remarkable medical or family history related to arterial hypertension, fibromuscular dysplasia or collagen disturbances, polycystic kidney disease, and homocystinuria. In addition, he denied tobacco smoking, alcohol abuse and illicit drug use. On admission, he had normal vital signs, and laboratory determinations including blood counts, coagulation tests, kidney and liver functions, blood glucose and lipid profile, were found within the normal ranges. On neurological examination, he was awake, alert, oriented; without language impairments, but with dysarthria; isochoric and photo reagent pupils, left eyelid ptosis, convergent strabismus, and horizontal nystagmus; left hemifacial hypoesthesia and hypoalgesia; fallen soft palate and decreased nausea reflex on the left side; muscular strength grade $I$ in the right lower limb, and grade II in the other limbs; symmetrical deep tendon reflexes $(2+)$ in upper limbs, asymmetrical reflexes in lower limbs (with right > left); bilateral absence of cutaneous plantar reflexes; and presence of ataxic gait, in addition to a Glasgow coma scale of 15 . Taken together, his history and physical findings were strongly indicative of ischemic stroke secondary to arterial dissection probably caused by neck and head trauma. Further imaging studies included cranial magnetic resonance imaging (MRI) and magnetic resonance angiography (MRA) of the neck, which showed consistent features of dissection at IV segment of the left vertebral artery and absence of blood flow related to basilar artery thrombosis. Additionally, 
there were characteristic features of ischemic stroke involving the left cerebellar hemisphere as well as the posterolateral bulbar region (Figure 1).

During two weeks of treatment, he evolved with gradual improvement of headache and his neurological disturbances recovered without complications or remarkable sequels. He was discharged to home undergoing physiotherapy sessions and uniquely taking aspirin $100 \mathrm{mg}$ daily. The patient has been performing normal daily and professional activities, and remains under clinical control in outpatient neurological service.

\section{Discussion}

In accordance with literature, this young patient presented with VAD, plus basilar artery thrombosis after a head trauma. Anatomical relationships between the vertebral arteries and spine may have favored the arterial dissection by stretching or compression, as has been described in forceful movements during neck hyperextension, rotation, flexion and distraction [1, 3]. VAD is considered an uncommon condition following blunt trauma and is observed in approximately $1 \%$ of the cases; nevertheless, this rate seems is probably underestimated $[1,4]$. Worthy of note, the variable asymptomatic period between the head trauma and initial symptoms of dissection can cause diagnostic pitfalls [1]. Headache in the occipital and temporal regions was the herald of the VAD in our patient, further followed by the neurological disturbances. Isolated thunderclap headache and excruciating neck pain have been described in young patients with diagnosis of VAD and delayed stroke [2]. Less common manifestations of VAD include generalized convulsive seizures [1], sensorineural hearing loss [5], and cervical radiculopathy [8].

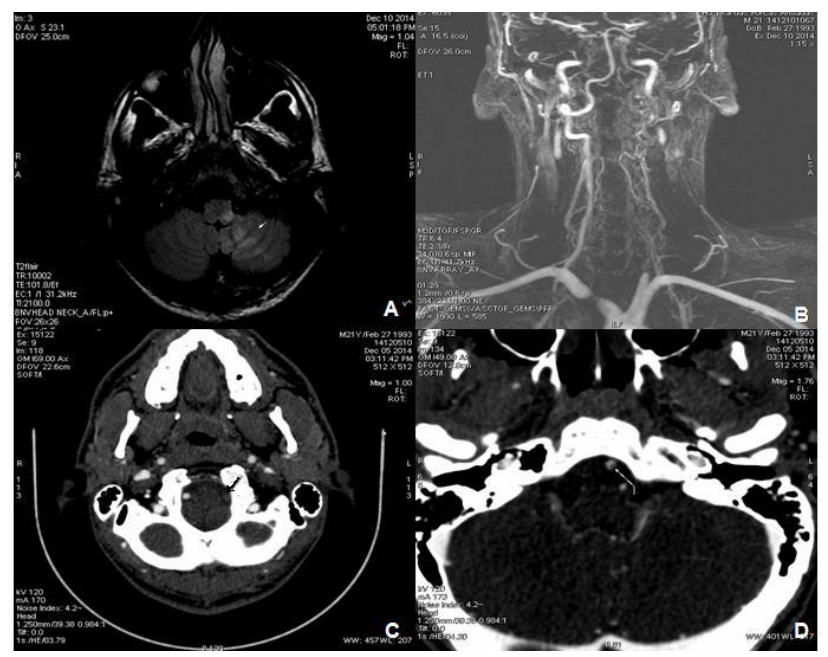

Figure 1. A: MRI axial flair weighted image of ischemic stroke affecting the left cerebellar hemisphere and the posterolateral bulbar region (white arrow); B: 3D-SPGR MRI images of normal blood flow in the right vertebral artery and absence of flow in the left vertebral artery; C: CT with contrast showing absence of blood flow in the left vertebral artery due to thrombosis (black arrow); D: CT with contrast showing a little thombus in the basilar artery (white arrow).
In addition to VAD, basilar artery thromboembolism was detected in the present case, and caused the classic presentation of this condition - initial headache and neck pain, and late development of ischemic cerebral symptoms [1, 2]. Dissections more often begin with subintimal hematoma, which causes a progressive occlusion and propitiate the origin of distal thrombosis [2]. Thromboembolic episodes are frequently reported complications of VAD, with increased risk of serious outcomes if the basilar artery is affected [1]. Notwithstanding, the patient of this case study underwent a conservative treatment with success, and the antiplatelet medication used was acetylsalicylic acid $100 \mathrm{mg}$ daily $[2,8]$.

As revealed by imaging studies, the post traumatic VAD herein commented, had developed in the V4 segment of the left vertebral artery. Kocaeli et al. described the main features of spontaneous intradural VAD and treatment strategies in 457 patients, predominantly males; the average age was 51.2 years, and the most common site of dissection was in the V2 and V3 segments of the artery. Different from traumatic VAD, the prognosis was poorer, subarachnoid hemorrhage occurred in $79 \%$ and was recurrent in $37 \%$ of the cases; and the main predisposing factors of spontaneous intradural VAD were arterial hypertension, fibromuscular dysplasia, and collagen abnormalities [9]. Hemorrhagic events were managed with invasive procedures, and ischemic strokes were treated with blood pressure control, antiplatelet or anticoagulant drugs [9].

The diagnosis in the present case may be clinically and radiologically characterized as Wallenberg syndrome or lateral medullary infarction, which was first described in 1895 [10-13]. Clinical characteristics of this syndrome are variable (as well as respective sites of damage), and may include: ipsilateral facial hemianalgesia (spinal trigeminal nucleus); ataxia and ipsilateral incoordination (lower cerebellar peduncle), dysarthria, dysphagia, decreased ipsilateral nausea reflex (nucleus ambiguus), ipsilateral Horner syndrome (oculosympathetic pathway); dizziness, nystagmus (vestibular nucleus); and contralateral thermal analgesia (lateral spinothalamic tract) [10-13]. More frequently, the syndrome involves injury in the lateral area, dorsal to the cuneiform bulbar olive, usually secondary to occlusion either of the vertebral artery or the posterior inferior cerebellar artery. Notwithstanding, it can have diverse origin as demyelinating injuries, aneurysm, abscess, and adverse effects of radiation.

On the clinical standpoint, the main differential diagnosis of VAD should include internal carotid artery dissection (iCAD), cervical spinal epidural hemorrhage, subarachnoid hemorrhage, cerebral venous thrombosis, and cerebrovascular constriction syndrome $[1-4,14,15]$. Santos et al. described a previously healthy 27-year-old woman with spontaneous iCAD, a condition which was considered a major differential hypothesis to explain the intense headache and neurological symptoms in the present case study. Spontaneous iCAD may occur in up to 3 per 100,000 people per year and cause ischemic strokes in patients with less than 50 years of age [14]. Comparative data between spontaneous ICAD and spontaneous VAD were described by von Babo et al. They reviewed findings from 1027 patients - 302 with VAD versus 668 with iCAD, and found some significant differences. The frequency of $\mathrm{iCAD}$ was two times higher than VAD and the frequency of bilateral dissection was two times higher in VAD group. Patients with iCAD were older and predominantly males; tobacco smoking predominated in VAD group [15]. Interestingly, pulsatile tinnitus 
occurred three times more often in cases of iCAD, and no difference was found in relation to antecedent infection or minor cervical trauma [15]. The embryological origin of the internal carotid artery is the same of the ascending aorta while the vertebral artery has the same embryological origin of the thoracic aorta; therefore, prospective studies might indicate more specific strategies for prevention and management of these two seemingly separate groups of dissection in cervical arteries [15]. Interestingly, Aronov et al., reported spontaneous quadruple cervical artery dissection, that is an exceeding rare condition with estimated incidence of 1 to 3 people per million of population [16]. Different of the present case, Yeh et al. described traumatic VAD and Wallemberg syndrome ten hours after a minor motorcycle accident [12].

Brain computed tomography $\mathrm{CT}$ and brain magnetic resonance imaging MRI, in addition to angiographic studies is indispensable tools for the establishment of correct diagnosis in patients with clinical suspicion of VAD or neck and head arterial thrombosis. Worthy of note, non contrasted CT scans sometimes appear initially normal $[1,6]$, an occurrence which may propitiate additional diagnostic pitfalls.

\section{Conclusion}

Primary health care workers should pay special attention about the hypothesis of cervical artery dissections in young individuals. This challenging condition may be spontaneous or occur unsuspected after minor trauma in the neck region during sport or common daily activities. Neurological symptoms may be initially absent and appear from hours to over than a week later, phenomenon related to diagnostic pitfalls. Delayed diagnosis plus late beginning of treatment can be associated with high risk of serious consequences; therefore, imaging studies of the neck and brain arteries must be utilized to establish the correct diagnosis and to control the improvement of the initial changes during treatment. Authors believe that case studies can contribute to increase the suspicion index of primary health care workers about vertebral artery dissection.

Conflict of interest: None to be declared.

\section{References}

1. Amin FM, Larsen VA, Tfelt-Hansen P. Vertebral artery dissection associated with generalized convulsive seizures: a case report. Case Rep Neurol 2013; 5(2): 125-129. (doi: 10.1159/000354033) (PMID: 23904852)

2. Hsu YC, Sung SF. Spontaneous vertebral artery dissection with thunderclap headache: a case report and review of the literature. Acta Neurol Taiwan 2014; 23(1): 24-28. (PMID: 24833212)

3. Menon RK, Norris JW. Cervical arterial dissection. Current concepts. Ann NY Acad Sci 2008; 1142: 200-217. (doi: 10.1196/annals.1444.015) (PMID: 18990128)

4. Redekop GJ. Extracranial carotid and vertebral artery dissection: a review. Can J Neurol Sci 2008; 34: 146-152. (PMID: 18574926)

5. Kim E, Son MK, Kang CK, Lee YB. Vertebrobasilar occlusion presenting as sudden isolated bilateral sensorineural hearing loss: case report. $J$ Cerebrovasc Endovasc Neurosurg 2013; 15(3): 225-228. (PMID: 24167804) (doi: 10.7461/jcen.2013.15.3.225)

6. Kwak YS, Kang DH, Woo HJ. Simultaneous vertebral artery dissection and contralateral posterior inferior cerebellar artery dissecting aneurysm. J Cerebrovasc Endovasc Neurosurg 2013; 14(3): 228-232. (doi: 10.746 I/jcen.2012.14.3.228)
7. Li S, Yan B, Kaye A, Mitchell P, Dowling R, Collins M, Davis S. Prognosis of intracranial dissection relates to site and presenting features. J Clin Neurosci 2011; 18(6): 789-793. (doi: 10.1016/j.jocn.2010.11.006) (PMID: 21507658)

8. Silbert $\mathrm{BI}$, Khangure $\mathrm{M}$, Silbert PL. Vertebral artery dissection as a cause of cervical radiculopathy. Asian Spine J 2013; 7(4): 335-338. (PMID: 24353851) (doi: 10.4184/asj.2013.7.4.335)

9. Kocaeli H, Chaalala C, Andaluz N, Zuccarello M. Spontaneous intradural vertebral artery dissection: a single-center experience and review of the literature. Skull Base 2009; 19(3): 209-218. (PMID: 19881901) (doi: 10.1055/s-0028-1114296)

10. Vrettos A, Fiotaki K, Galati E, Plachouras D. A crossed brain stem syndrome without crossed sensory symptomatology. BMJ Case Rep 2013. (doi: 10.1136/bcr-2012-006709)

11. Wallenberg A. Acute Bulbäraffection (Embolie der Art. Cerebellar post. inf. sinistr.?). Arch Psychiatr Nervenheilkd 1895; 27: 504-540.

12. Yeh HF, Seak CJ, Chiu TF, Chang YC. Traumatic vertebral artery dissection and Wallenberg syndrome after a motorcycle collision. Am J Emerg Med 2009; 27(1): 131.e1-3. (doi: 10.1016/j.ajem.2008.04.025)

13. Zhang SQ, Liu MY, Wan B, Zheng HM. Contralateral body half hypalgesia in a patient with lateral medullary infarction: atypical Wallemberg syndrome. Eur Neurol 2008; 59(3-4): 211-215. (doi: 10.1159/000114050)

14. Santos VM, Santos AMRO, Ribeiro KRA, Ribeiro KD, Silva FHO, Marquezini ECBM. Spontaneous dissection of the internal carotid artery in a young patient: case report. Brasilia Med 2013; 50(3): 266270. (doi: 10.14242/2236-5117.2014av50n3a99p266)

15. von Babo M, De Marchis GM, Sarikaya H, Stapf C, Buffon F, Fischer U, et al. Differences and similarities between spontaneous dissections of the lateral carotid artery and the vertebral artery. Stroke 2013; 44(6): 1537-1542. (PMID: 23632978) (doi: 10.1161/strokeaha.113.001057)

16. Aronov M, Shevchenko NS, Amosova NA, Kotenko KV. Acute threevessel cervical arterial occlusion due to spontaneous quadruple cervical artery dissection. BMJ Case Rep 2014; 2014. (doi: 10.1136/bcr2014-203725)

\section{Authors:}

Vitorino Modesto dos Santos - MD, PhD, Professor of Internal Medicine, Catholic University and Armed Forces Hospital, Brasília-DF, Brazil.

Ursula Valeska Poti Araújo Lima - MD, Neurologist, Armed Forces Hospital, Brasília-DF, Brazil.

Renata Faria Silva - MD, Internal Medicine, Armed Forces Hospital, Brasília-DF, Brazil.

Silvana Alves Gomma de Azevedo - MD, Radiologist, Armed Forces Hospital, Brasília-DF, Brazil.

Melissa Gebrim Ribeiro - MD, Internal Medicine, Armed Forces Hospital, Brasília-DF, Brazil.

Daniela Gomes Gebrim - MD, Internal Medicine, Armed Forces Hospital, Brasília-DF, Brazil. 